\title{
Identify Key Active Ingredients and Molecular Mechanisms of Jiangzhi Decoction on Non- alcoholic Fatty Liver Disease by Network Pharmacology Analysis
}

\section{Lei Wang}

Shanghai Seventh People's Hospital

\section{Yin Zhi}

Shanghai Seventh People's Hospital

\section{Ying Ye}

Shanghai Seventh People's Hospital

\section{Miao Zhang}

Shanghai Seventh People's Hospital

\section{Xing Ma}

Shanghai Seventh People's Hospital

Hongyun Tie

Shanghai Seventh People's Hospital

Wei Xia

Shanghai Seventh People's Hospital

Yanan Song ( $\nabla$ synabc.123@163.com )

Shanghai Seventh People's Hospital

\section{Research}

Keywords: non-alcoholic fatty liver disease (NAFLD), Jiangzhi Decoction (JZD), network pharmacology, protein-protein interaction (PPI), ReactomeFIViz, ClueGO

Posted Date: June 18th, 2020

DOl: https://doi.org/10.21203/rs.3.rs-35476/v1

License: (c) (1) This work is licensed under a Creative Commons Attribution 4.0 International License. Read Full License 


\section{Abstract}

Background: Jiangzhi Decoction (JZD), a traditional herb mixture, has shown significant clinical efficacy against non-alcoholic fatty liver disease (NAFLD). However, its multicomponent and multitarget characteristics bring difficulty in deciphering its pharmacological mechanisms. Our study aimed to identify the key active ingredients and core molecular mechanisms of JZD against NAFLD.

Methods: The active ingredients were searched from Traditional Chinese Medicine Systems Pharmacology (TCMSP) database and Traditional Chinese Medicine Integrated Database (TCMID). The targets of those ingredients were identified using ChemMapper database based on 3D-structure similarity. NAFLD-related genes were searched from DisGeNET database and Gene Expression Omnibus (GEO) database. Then we obtained candidate targets of JZD against NAFLD by overlapping the active ingredient targets and NAFLD-related genes. We performed protein-protein interaction (PPI) analysis, functional enrichment analysis and constructed pathway networks of "herbs-active ingredients-candidate targets", and identified the key active ingredients and core molecular mechanisms in the network.

Results: We found 147 active ingredients in JZD, 1285 targets of active ingredients, 401 NAFLD-related genes, and 59 overlapped candidate targets of JZD against NAFLD. 22 core targets were obtained by PPI analysis. Finally, nuclear receptor transcription and lipid metabolism regulation were found as the core molecular mechanisms of JZD against NAFLD by functional enrichment analysis, and emodin, emodin anthrone, hyperin, questin, rhein, etc. were speculated as the key active ingredients of JZD.

Conclusion: Our study will provide the scientific evidences of the clinical efficacy of JZD against NAFLD.

\section{Introduction}

Non-alcoholic fatty liver disease (NAFLD) encompasses a spectrum of liver pathology that is characterized by the excessive accumulation of fat in the liver, including simple steatosis non-alcoholic fatty liver (NAFL), non-alcoholic steatohepatitis (NASH), steatofibrosis, cirrhosis, and hepatocellular carcinoma [1]. In the face of a global obesity epidemic, NAFLD has emerged as the most common form of chronic liver disease, affecting an estimated $25 \%$ of the general population worldwide [2,3]. Epidemiological researches have reported that NAFLD is one of the three main causes of cirrhosis [4], and $\mathrm{NASH}$ is rapidly becoming the leading cause of end-stage liver disease $[5,6]$. However, except for lifestyle interventions, such as exercise and dietary, there is still no approved pharmacotherapy for NAFLD. Therefore, it is important to explore and develop valid pharmacotherapies for NAFLD [7].

In recent years, traditional Chinese medicine (TCM) has been proved effective in the case of alone or integrated with western medicine, and has attracted more and more people's attention [8, 9]. Jiangzhi Decoction (JZD), a clinically used herbal formula developed in accordance with TCM pathogenesis, is composed of the following five medicinal herbs: Trichosanthes Kirilowii (TK), Alisma Orientale (AO), Angelica Sinensis (AS), Crataegus Pinnatifida (CP) and Polygonum Multiflorum (PM). Previous evidence 
has proved the efficacy of JZD on regulating lipid metabolism [10]. However, the key active ingredients and molecular mechanisms of JZD are still unclear and need further exploration.

Because Chinese herbal medicines have the characteristics of multi-component and multi-targeted effects, conventional strategies can be hardly used to explore their pharmacological mechanisms. Network pharmacology, a novel approach based on systems biology, has been proved suitable for analyzing the complex relationships of various ingredients and effects in Chinese herbal medicines [11]. In this study, network pharmacology was carried out to investigate the key active ingredients and molecular mechanisms according to screening a great deal of candidate ingredients, predicting multiple drug targets, analyzing possible signaling pathways and conducting herbs-ingredients-targets networks. A flowchart of the network pharmacology approach is presented in Fig. 1.

\section{Methods}

\subsection{Searching for active ingredients of JZD}

Active ingredients of JZD were collected from two databases. One is Traditional Chinese Medicine Systems Pharmacology (TCMSP) database [12] (http://lsp.nwu.edu.cn/tcmsp.php), which contains large number of herbal entries, drug-disease networks and drug-target networks. A great deal of herbal information can be obtained from TCMSP database, including ingredients, molecule name, molecular weight $(\mathrm{MW})$, drug-likeness $(\mathrm{DL})$, human oral bioavailability $(\mathrm{OB})$, half-life $(\mathrm{HL})$, water partition coefficient (AlogP), number of hydrogen bond donors and receptors (Hdon/Hacc), Caco-2 permeability (Caco-2), blood-brain barrier (BBB) and so on. The active ingredients of JZD were screened out according to the $A D M E$ parameter, and the ingredients with $D L \geq 0.18$ were regarded as active ingredients [13].

If the herbal information could not be found in TCMSP database, the other database would be used. It's Traditional Chinese Medicine Integrated Database (TCMID) (http://www.megabionet.org/tcmid/), which contains 46929 prescriptions, 8159 herbs, 43413 total ingredients, 8182 drugs, 4633 diseases, 1045 prescription ingredients, 778 herbal mass spectra, 3895 mass spectrometry of ingredients [14]. By combining the information from two databases above, the active ingredients of JZD were identified.

\subsection{Identification of targets of active ingredients}

ChemMapper database (http://lilab.ecust.edu.cn/chemmapper/) is a versatile web server for exploring pharmacology and chemical structure association based on molecular 3D similarity method [15]. We searched the predicted targets of each active ingredient in JZD from ChemMapper database and screened according to the criteria of 3D structure similarity above 1.0 and prediction score above 0 [16]. The full names of targets were converted to gene symbol based on the UniProt ID in UniProt database (http://www.uniprot.org/) for further analysis.

\subsection{Searching for NAFLD-related genes}


DisGeNET database (http://www.disgenet.org/web/DisGeNET/menu/home) is a knowledge management platform integrating and standardizing data about disease associated genes and variants from multiple sources, including the scientific literature [17]. Known genes of NAFLD were searched from DisGeNET database using "non-alcoholic fatty liver disease" as the keyword, and the top $30 \%$ of genes were regarded as important genes for further analysis.

In addition, Gene Expression Omnibus (GEO) database (http://www.ncbi.nlm.nih.gov/geo/) is an international public repository for high-throughput microarray and next-generation sequence functional genomic data sets submitted by the research community [18]. The main differentially expressed genes (DEGs) between mild NAFLD and advanced NAFLD were extracted from microarray data GSE31803 and GSE49541 in GEO database, with a cutoff value of $P<0.05$ and fold change $|\mathrm{FC}| \geq 3$.

\subsection{Protein-protein interaction (PPI) analysis}

The overlapped genes between the target genes of active ingredients and NAFLD-related genes were imported into STRING database to construct PPI network. STRING database integrates the qualitycontrolled protein-protein association networks of a large number of organisms. We selected the core PPI targets according to the degree score above the average value and the confidence score above 0.9 .

\subsection{Functional enrichment analysis}

ReactomeFIViz and ClueGO, two kinds of plug-ins for Cytoscape, were used to perform functional enrichment analysis. ReactomeFIViz is a highly reliable protein functional interaction network covering around $60 \%$ of total human genes based on Reactome database, the most popular and comprehensive open-source biological pathway knowledgebase [19]. ClueGO integrates Gene Ontology (GO) terms and KEGG/BioCarta pathways, and creates a functionally organized GO/pathway term network [20]. $P<0.01$ was regarded as the significant cutoff in this study.

\subsection{Pathway network construction}

Pathway networks of "herbs-active ingredients-candidate targets" were constructed using the Cytoscape 3.3.0 software. The Network Analyzer plug-in was used to identify key active ingredients and critical candidate targets based on the criterion below: nodes with degree values exceeding the average value of all nodes in the network. The degree value is the number of edges a node has in a network, which indicates how many herbs/ingredients/targets one node is related with. If the degree value of a node is larger, the node is believed to play a more important role in the network.

\section{Results}

\subsection{Active ingredients of JZD}

Among the five main herbs of JZD, TK, AO and AS were searched from TCMSP database, while the other herbs, CP and PM, couldn't be found in TCMSP database and were searched from TCMID database. Based on the criteria of $D L \geq 0.18$, a total of 147 active ingredients were finally screened out in this study 
(Fig. 2 and Supplementary Table 1). The numbers of active ingredients in TK, AO, AS, CP and PM were 17, 15, 7, 66 and 46, respectively. There were four ingredients overlapped in two herbs, including emodin in $\mathrm{AO}$ and $\mathrm{PM}$, epicatechin in $\mathrm{CP}$ and $\mathrm{PM}$, tricin in TK and $\mathrm{PM}$, and $\beta$-sitosterol in AS and PM.

A note about those results was that ADME parameters were not provided in TCMID database. Thus, the ingredients in CP and PM didn't be filtered according to the ADME parameters. Maybe it was the reason why the numbers of ingredients in CP and PM were much larger than those in TK, AO and AS.

\subsection{Targets of active ingredients of JZD}

The direct target of each chemical ingredient in JZD were identified from ChemMapper database. According to the criteria of 3D structure similarity above 1.0 and prediction score above 0 , a total of 1285 targets of active ingredients of JZD were obtained (Supplementary Table 2). Our analysis showed that gallic acid, citric acid and succinicacid were the top three active ingredients targeting 525, 481 and 440 targets, respectively.

\subsection{Genes related to NAFLD}

A total of 333 known genes were found from DisGeNET database, and the top 30\%, that were 100 genes, were chosen for further analysis. Based on the cutoff value of $P<0.05$ and fold change $|\mathrm{FC}| \geq 3$, a total of 315 DEGs were extracted from microarray data GSE31803 and GSE49541. After duplicates of genes from DisGeNET database and GEO data were eliminated, a total of 401 genes were identified as NAFLD-related genes (Supplementary Table 3).

\subsection{Core targets in protein-protein interaction (PPI)}

According to the search and analysis above, we obtained a total of 59 overlapped genes between the target genes of active ingredients and NAFLD-related genes (Table 1). The 59 candidate targets were imported into STRING database to construct the PPI network. According to the screening criteria of the degree score above the average value and the confidence score above 0.9, 22 core targets were obtained, including TNF, IGF1, IL1B, GPT, CCL2, HGF, TLR4, PPARG, GOT2, LDLR, MMP1, F2, DCN, ACE, GLUL, PPARA, THBS1, JAK2, CFTR, PLAT, OAT and GPX8 (Fig. 3). The 22 core targets may be the potential targets in the treatment of JZD against NAFLD. 
Table 1

The overlapped genes between the target genes of active ingredients and NAFLD-related genes

\begin{tabular}{|c|c|c|c|c|}
\hline No. & $\begin{array}{l}\text { Uniprot } \\
\text { ID }\end{array}$ & $\begin{array}{l}\text { Gene } \\
\text { ID }\end{array}$ & $\begin{array}{l}\text { Gene } \\
\text { Symbol }\end{array}$ & Gene Full Name \\
\hline 1 & P54710 & 486 & FXYD2 & FXYD Domain Containing Ion Transport Regulator 2 \\
\hline 2 & P06396 & 2934 & GSN & Gelsolin \\
\hline 3 & P28472 & 2562 & GABRB3 & $\begin{array}{l}\text { Gamma-Aminobutyric Acid Type A Receptor Beta3 } \\
\text { Subunit }\end{array}$ \\
\hline 4 & P07996 & 7057 & THBS1 & Thrombospondin 1 \\
\hline 5 & P54289 & 781 & CACNA2D1 & $\begin{array}{l}\text { Calcium Voltage-Gated Channel Auxiliary Subunit } \\
\text { Alpha2delta } 1\end{array}$ \\
\hline 6 & P04818 & 7298 & TYMS & Thymidylate Synthetase \\
\hline 7 & P17302 & 2697 & GJA1 & Gap Junction Protein Alpha 1 \\
\hline 8 & P55011 & 6558 & SLC12A2 & Solute Carrier Family 12 Member 2 \\
\hline 9 & P07585 & 1634 & DCN & Decorin \\
\hline 10 & P08729 & 3855 & KRT7 & Keratin 7 \\
\hline 11 & P14210 & 3082 & HGF & Hepatocyte Growth Factor \\
\hline 12 & P13569 & 1080 & CFTR & CF Transmembrane Conductance Regulator \\
\hline 13 & Q96SL4 & 2882 & GPX7 & Glutathione Peroxidase 7 \\
\hline 14 & P13500 & 6347 & CCL2 & C-C Motif Chemokine Ligand 2 \\
\hline 15 & 094925 & 2744 & GLS & Glutaminase \\
\hline 16 & P21802 & 2263 & FGFR2 & Fibroblast Growth Factor Receptor 2 \\
\hline 17 & Q99584 & 6284 & S100A13 & S100 Calcium Binding Protein A13 \\
\hline 18 & Q13133 & 10062 & $\mathrm{NR} 1 \mathrm{H} 3$ & Nuclear Receptor Subfamily 1 Group H Member 3 \\
\hline 19 & P13716 & 210 & ALAD & Aminolevulinate Dehydratase \\
\hline 20 & P82251 & 11136 & SLC7A9 & Solute Carrier Family 7 Member 9 \\
\hline 21 & Q8N159 & 162417 & NAGS & N-Acetylglutamate Synthase \\
\hline 22 & P21549 & 189 & AGXT & $\begin{array}{l}\text { Alanine-Glyoxylate And Serine-Pyruvate } \\
\text { Aminotransferase }\end{array}$ \\
\hline 23 & P34896 & 6470 & SHMT1 & Serine Hydroxymethyltransferase 1 \\
\hline 24 & Q08828 & 107 & ADCY1 & Adenylate Cyclase 1 \\
\hline 25 & 043708 & 2954 & GSTZ1 & Glutathione S-Transferase Zeta 1 \\
\hline
\end{tabular}




\begin{tabular}{|c|c|c|c|c|}
\hline No. & $\begin{array}{l}\text { Uniprot } \\
\text { ID }\end{array}$ & $\begin{array}{l}\text { Gene } \\
\text { ID }\end{array}$ & $\begin{array}{l}\text { Gene } \\
\text { Symbol }\end{array}$ & Gene Full Name \\
\hline 26 & P51570 & 2584 & GALK1 & Galactokinase 1 \\
\hline 27 & P15104 & 2752 & GLUL & Glutamate-Ammonia Ligase \\
\hline 28 & Q14749 & 27232 & GNMT & Glycine N-Methyltransferase \\
\hline 29 & P36222 & 1116 & CHI3L1 & Chitinase 3 Like 1 \\
\hline 30 & Q8TED1 & 493869 & GPX8 & Glutathione Peroxidase 8 (Putative) \\
\hline 31 & P11137 & 4133 & MAP2 & Microtubule Associated Protein 2 \\
\hline 32 & P00750 & 5327 & PLAT & Plasminogen Activator, Tissue Type \\
\hline 33 & Q9H2S1 & 3781 & KCNN2 & $\begin{array}{l}\text { Potassium Calcium-Activated Channel Subfamily N } \\
\text { Member } 2\end{array}$ \\
\hline 34 & P04181 & 4942 & OAT & Ornithine Aminotransferase \\
\hline 35 & Q07869 & 5465 & PPARA & Peroxisome Proliferator Activated Receptor Alpha \\
\hline 36 & P01130 & 3949 & LDLR & Low Density Lipoprotein Receptor \\
\hline 37 & P09488 & 2944 & GSTM1 & Glutathione S-Transferase Mu 1 \\
\hline 38 & P09211 & 2950 & GSTP1 & Glutathione S-Transferase Pi 1 \\
\hline 39 & Q03181 & 5467 & PPARD & Peroxisome Proliferator Activated Receptor Delta \\
\hline 40 & P30711 & 2952 & GSTT1 & Glutathione S-Transferase Theta 1 \\
\hline 41 & P08263 & 2938 & GSTA1 & Glutathione S-Transferase Alpha 1 \\
\hline 42 & P03956 & 4312 & MMP1 & Matrix Metallopeptidase 1 \\
\hline 43 & Q9P2W7 & 27087 & B3GAT1 & Beta-1,3-Glucurony/transferase 1 \\
\hline 44 & 060674 & 3717 & JAK2 & Janus Kinase 2 \\
\hline 45 & P08700 & 3562 & IL3 & Interleukin 3 \\
\hline 46 & P12821 & 1636 & ACE & Angiotensin I Converting Enzyme \\
\hline 47 & P00734 & 2147 & $\mathrm{~F} 2$ & Coagulation Factor II, Thrombin \\
\hline 48 & Q8WTV0 & 949 & SCARB1 & Scavenger Receptor Class B Member 1 \\
\hline 49 & P06576 & 506 & ATP5F1B & ATP Synthase F1 Subunit Beta \\
\hline 50 & 014594 & 1463 & NCAN & Neurocan \\
\hline 51 & P24298 & 2875 & GPT & Glutamic-Pyruvic Transaminase \\
\hline 52 & P01375 & 7124 & TNF & Tumor Necrosis Factor \\
\hline
\end{tabular}




\begin{tabular}{|cllll|}
\hline No. & $\begin{array}{l}\text { Uniprot } \\
\text { ID }\end{array}$ & $\begin{array}{l}\text { Gene } \\
\text { ID }\end{array}$ & $\begin{array}{l}\text { Gene } \\
\text { Symbol }\end{array}$ & Gene Full Name \\
\hline 53 & P37231 & 5468 & PPARG & Peroxisome Proliferator Activated Receptor Gamma \\
\hline 54 & 000206 & 7099 & TLR4 & Toll Like Receptor 4 \\
\hline 55 & P06213 & 3643 & INSR & Insulin Receptor \\
\hline 56 & P00505 & 2806 & GOT2 & Glutamic-Oxaloacetic Transaminase 2 \\
\hline 57 & P01584 & 3553 & IL1B & Interleukin 1 Beta \\
\hline 58 & P05019 & 3479 & IGF1 & Insulin Like Growth Factor 1 \\
\hline 59 & P41235 & 3172 & HNF4A & Hepatocyte Nuclear Factor 4 Alpha \\
\hline
\end{tabular}

\subsection{Functional enrichment analysis of the overlapped genes}

ReactomeFIViz and ClueGO pathway analyses were further performed for the overlapped genes. The top 10 significantly related pathways were shown in Fig. 4A by ReactomeFIViz analysis, including nuclear receptor transcription pathway, glutathione conjugation, interleukin-4 and 13 signaling, regulation of insulin-like growth factor (IGF) transport and uptake by insulin-like growth factor binding proteins (IGFBPs), phase II conjugation and so on. At the same time, according to ClueGo analysis, signaling pathways were divided into 13 enriched categories based the kappa coefficient, including positive regulation of lipid metabolic process, regulation of smooth muscle cell proliferation, fatty acid transport, positive regulation of reactive oxygen species metabolic process, regulation of phosphatidylinositol 3kinase signaling, alpha-amino acid metabolic process, regeneration, response to mechanical stimulus, muscle cell proliferation, cellular detoxification, exocrine system development, regulation of glucose transport, and cell-cell signaling involved in cardiac conduction (Fig. 4B and 4C). The functional enrichment analysis indicated that nuclear receptor transcription and lipid metabolism regulation might play an important role in the treatment of JZD against NAFLD.

\subsection{Pathway network construction of herb-ingredient-target}

To identify key active ingredients and molecular mechanisms of JZD against NAFLD, we further constructed herb-ingredient-target networks based on the top significantly related pathways from functional enrichment analysis (Fig. 5). We found that 12 ingredients appeared in both nuclear receptor transcription pathway and lipid metabolism regulation pathway, including emodin, emodin anthrone, hyperin, questin, rhein, tricin, aloe emodin, 2-acetylemodin, epicatechin, chrysazin, chrysophanol, 4,4'dihydroxydiphenyl methane. The results implied that those key active ingredients all participated in nuclear receptor transcription and lipid metabolism regulation, which might be the key molecular mechanisms of JZD against NAFLD.

\section{Discussion}


NAFLD is characterized by abnormal lipid metabolism and excessive lipid accumulation in hepatocytes. Most of the herbs in JZD have been reported to take part in the regulation of lipid metabolism in NAFLD or other related diseases. Some researches showed that Alisma Orientale (AO) could prevent hepatic triglyceride accumulation through suppressing de novo lipogenesis and increasing lipid export, and control oxidative stress markers, lipoapoptosis, liver injury panels and inflammatory and fibrotic mediators, eventually influencing steatohepatitis and liver fibrosis [21,22]. The components of Angelica Sinensis (AS) have been proved to regulate lipid and glucose metabolism [23-25]. Liu et al. found that a diet formula of Crataegus Pinnatifida (CP) and three other herbs could alleviate hepatic steatosis and insulin resistance in vivo and in vitro [26]. Yu et al. performed a serious of experiments to confirm that the active components of Polygonum Multiflorum (PM) could promote the lipolysis of cholesterol and triglyceride, increase the content of HTGL, and reduce LDL and VLDL [27-29]. However, the synergetic mechanisms of all the herbs in JZD were still unclear.

In our study, nuclear receptor transcription and lipid metabolism regulation were found as the core pathways which JZD mainly participated in when alleviating NAFLD. As we know, there are 48 nuclear receptors categorized into 7 subfamilies designated as NR0-NR6 [30]. Of particular importance in NAFLD are specific members of NR1 subfamily [31]. Most potential targets of JZD in Fig. 2 belong to NR1 subfamily, including PPARs (peroxisome proliferator-activated receptors, PPARA/PPARa, PPARD/PPAR $\beta$ / ס, PPARG/PPARY; NR1C1-3) and LXRa (liver $X$ receptor a; NR1H3). PPARa activation induces the increase in fatty acid oxidation, ketogenesis and gluconeogenesis [32]. PPAR $/ \delta$ activation exerts regulatory effects on fatty acid catabolism, reverse cholesterol transport and energy metabolism, and even reduces insulin resistance and plasma glucose [33]. PPARy shifts lipids from non-adipose organs such as the liver and skeletal muscles to white adipose tissue, leading to the attenuation of lipotoxicity [34]. In general, PPAR activation is thought to be beneficial in NAFLD, and clinical trials of single/dual receptor agonists are underway [31]. Another important nuclear receptor LXRa acts as the negative regulator of cholesterol metabolism through the induction of hepatocyte cholesterol catabolism, excretion, and the reverse cholesterol transport pathway [35]. Furthermore, HNF4A/HNF4a (hepatocyte nuclear factor 4a; NR2A1) also belongs to the subfamily of nuclear receptors. Previous study reported that HNF4a could prevent liver steatosis by controlling hepatic carboxylesterase 2 expression and modulating lipolysis, lipogenesis and endoplasmic reticulum in NAFLD [36]. Therefore, they are all potential therapeutic targets for the treatment of NAFLD.

Our research found that some ingredients in JZD might be the key ones for NAFLD treatment, such as emodin, hyperin and rhein. Many previous studies have reported that those ingredients could take part in the regulation of nuclear receptor. Emodin has been proved to increase the mRNA level of PPARY and play a protective role in alcohol-mediated liver steatosis [37]. According to the activation of PPARY signaling pathway, emodin could also alleviate atherosclerosis followed by promoting cholesterol efflux [38], or play other roles though regulating inflammatory response $[39,40]$ and nitric oxide production [41]. Furthermore, emodin has also been reported to regulate the expression of LXRa in atherosclerosis [38] and melanogenesis [42]. Hyperin is one of the chief flavonoid components of Ericaceae, Guttifera, Leguminosae and Celastraceae, and could remarkably induce the expression of PPARy and attenuate 
inflammation of acute liver injury [43]. In addition, some studies also reported that rhein could target PPARY signaling pathway and play anti-inflammatory activity $[44,45]$. Moreover, rhein has been confirmed to ameliorate NAFLD and obesity and recover metabolic disorders through directly binding to LXRa $[46,47]$. Thus, those key ingredients in JZD might improve NAFLD via regulating the nuclear receptors.

In conclusion, the multicomponent and multitarget characteristics of the therapeutic effects of JZD against NAFLD were effectively elucidated through network pharmacology approach. Emodin, emodin anthrone, hyperin, questin, rhein were speculated as the key active ingredients of JZD, and nuclear receptor transcription and lipid metabolism regulation were found as the core molecular mechanisms by which JZD alleviated NAFLD (Fig. 6). Therefore, our study will provide the scientific evidences of the clinical efficacy of JZD against NAFLD.

\section{Declarations}

\section{Ethics approval and consent to participate}

Not applicable.

\section{Consent for publication}

Not applicable.

\section{Availability of data and materials}

The data of our research can be acquired from the Supplementary Materials uploaded with this article.

\section{Competing interests}

The authors declare that they have no competing interests.

\section{Funding}

The study was supported by grants from National Natural Science Foundation of China (No. 81703791, No. 81873178 and No. 81904036), Shanghai Municipal Health Commission (No.201740318), Science and Technology Development Fund of Shanghai Pudong New Area (No. PKJ2017-Y13), Talents Training Program of Pudong Health Commission of Shanghai (No. PWRq2017-02), and Talents Training Program of Seventh People's Hospital of Shanghai University of Traditional Chinese Medicine (No. XX2017-04 and No. XX2019-01). 


\section{Authors' contributions}

Song YN and Xia W conceived the study. Wang L, Zhi Y, Zhang M and Ma X analyzed the data. Zhi $Y$ and Tie HY made the charts. Wang $L$ wrote the paper. Song YN and Xia W revised the paper. Ye Y provided technical support.

\section{Acknowledgements}

Not applicable.

\section{References}

1. Younossi ZM (2019) Non-alcoholic fatty liver disease - A global public health perspective. J Hepatol 70: 531-544.

2. Younossi ZM, Koenig AB, Abdelatif D, Fazel Y, Henry L, et al. (2016) Global epidemiology of nonalcoholic fatty liver disease-Meta-analytic assessment of prevalence, incidence, and outcomes. Hepatology 64: 73-84.

3. Sanyal AJ (2019) Past, present and future perspectives in nonalcoholic fatty liver disease. Nat Rev Gastroenterol Hepatol 16: 377-386.

4. Zheng Y, Wang M, Zheng P, Tang X, Ji G (2018) Systems pharmacology-based exploration reveals mechanisms of anti-steatotic effects of Jiang Zhi Granule on non-alcoholic fatty liver disease. Sci Rep 8: 13681.

5. Marengo A, Jouness RI, Bugianesi E (2016) Progression and Natural History of Nonalcoholic Fatty Liver Disease in Adults. Clin Liver Dis 20: 313-324.

6. Goldberg D, Ditah IC, Saeian K, Lalehzari M, Aronsohn A, et al. (2017) Changes in the Prevalence of Hepatitis C Virus Infection, Nonalcoholic Steatohepatitis, and Alcoholic Liver Disease Among Patients With Cirrhosis or Liver Failure on the Waitlist for Liver Transplantation. Gastroenterology 152: 1090-1099 e1091.

7. Sumida Y, Yoneda M (2018) Current and future pharmacological therapies for NAFLD/NASH. J Gastroenterol 53: 362-376.

8. Liu Q, Zhu L, Cheng C, Hu YY, Feng Q (2017) Natural Active Compounds from Plant Food and Chinese Herbal Medicine for Nonalcoholic Fatty Liver Disease. Curr Pharm Des 23: 5136-5162.

9. Hsu WF, Sheen LY, Lin HJ, Chang HH (2016) A Review of Western and Traditional Chinese Medical Approaches to Managing Nonalcoholic Fatty Liver Disease. Evid Based Complement Alternat Med 2016: 6491420.

10. Li K, Li YJ, Zhang ZQ (2016) Study of Jiangzhi decoction on lipid metabolism. Journal of Henan Institute of Education (Natural Science Edition) 25: 35-37. 
11. Zhang R, Zhu X, Bai H, Ning K (2019) Network Pharmacology Databases for Traditional Chinese Medicine: Review and Assessment. Front Pharmacol 10: 123.

12. Ru J, Li P, Wang J, Zhou W, Li B, et al. (2014) TCMSP: a database of systems pharmacology for drug discovery from herbal medicines. J Cheminform 6: 13.

13. Gao L, Wang XD, Niu YY, Duan DD, Yang X, et al. (2016) Molecular targets of Chinese herbs: a clinical study of hepatoma based on network pharmacology. Sci Rep 6: 24944.

14. Huang L, Xie D, Yu Y, Liu H, Shi Y, et al. (2018) TCMID 2.0: a comprehensive resource for TCM. Nucleic Acids Res 46: D1117-D1120.

15. Gong J, Cai C, Liu X, Ku X, Jiang H, et al. (2013) ChemMapper: a versatile web server for exploring pharmacology and chemical structure association based on molecular 3D similarity method. Bioinformatics 29: 1827-1829.

16. Yu M, Song X, Yang W, Li Z, Ma X, et al. (2019) Identify the Key Active Ingredients and Pharmacological Mechanisms of Compound XiongShao Capsule in Treating Diabetic Peripheral Neuropathy by Network Pharmacology Approach. Evid Based Complement Alternat Med 2019: 5801591.

17. Pinero J, Ramirez-Anguita JM, Sauch-Pitarch J, Ronzano F, Centeno E, et al. (2019) The DisGeNET knowledge platform for disease genomics: 2019 update. Nucleic Acids Res.

18. Barrett T, Wilhite SE, Ledoux P, Evangelista C, Kim IF, et al. (2013) NCBI GEO: archive for functional genomics data sets-update. Nucleic Acids Res 41: D991-995.

19. Wu G, Haw R (2017) Functional Interaction Network Construction and Analysis for Disease Discovery. Methods Mol Biol 1558: 235-253.

20. Bindea G, Mlecnik B, Hackl H, Charoentong P, Tosolini M, et al. (2009) ClueGO: a Cytoscape plug-in to decipher functionally grouped gene ontology and pathway annotation networks. Bioinformatics 25 : 1091-1093.

21. Choi E, Jang E, Lee JH (2019) Pharmacological Activities of Alisma orientale against Nonalcoholic Fatty Liver Disease and Metabolic Syndrome: Literature Review. Evid Based Complement Alternat Med 2019: 2943162.

22. Shu Z, Pu J, Chen L, Zhang Y, Rahman K, et al. (2016) Alisma orientale: Ethnopharmacology, Phytochemistry and Pharmacology of an Important Traditional Chinese Medicine. Am J Chin Med 44: 227-251.

23. Wang K, Cao P, Shui W, Yang Q, Tang Z, et al. (2015) Angelica sinensis polysaccharide regulates glucose and lipid metabolism disorder in prediabetic and streptozotocin-induced diabetic mice through the elevation of glycogen levels and reduction of inflammatory factors. Food Funct 6: 902909.

24. Wang K, Cao P, Wang H, Tang Z, Wang N, et al. (2016) Chronic administration of Angelica sinensis polysaccharide effectively improves fatty liver and glucose homeostasis in high-fat diet-fed mice. Sci Rep 6: 26229. 
25. Zhong T, Zhang H, Duan X, Hu J, Wang L, et al. (2017) Anti-obesity effect of radix Angelica sinensis and candidate causative genes in transcriptome analyses of adipose tissues in high-fat diet-induced mice. Gene 599: 92-98.

26. Liu J, Zhang H, Ji B, Cai S, Wang R, et al. (2014) A diet formula of Puerariae radix, Lycium barbarum, Crataegus pinnatifida, and Polygonati rhizoma alleviates insulin resistance and hepatic steatosis in CD-1 mice and HepG2 cells. Food Funct 5: 1038-1049.

27. Wang M, Zhao R, Wang W, Mao X, Yu J (2012) Lipid regulation effects of Polygoni Multiflori Radix, its processed products and its major substances on steatosis human liver cell line L02. J Ethnopharmacol 139: 287-293.

28. Wang W, He Y, Lin P, Li Y, Sun R, et al. (2014) In vitro effects of active components of Polygonum Multiflorum Radix on enzymes involved in the lipid metabolism. J Ethnopharmacol 153: 763-770.

29. Lin P, Lu JM, Wang YF, Gu W, Zhao RH, et al. (2017) Prevention Mechanism of 2,3,5,4'-Tetrahydroxystilbene-2-0-beta-D-glucoside on Lipid Accumulation in Steatosis Hepatic L-02 Cell. Pharmacogn Mag 13: 245-253.

30. Evans RM, Mangelsdorf DJ (2014) Nuclear Receptors, RXR, and the Big Bang. Cell 157: 255-266.

31. Cave MC, Clair HB, Hardesty JE, Falkner KC, Feng W, et al. (2016) Nuclear receptors and nonalcoholic fatty liver disease. Biochim Biophys Acta 1859: 1083-1099.

32. Goto T (2019) A review of the studies on food-derived factors which regulate energy metabolism via the modulation of lipid-sensing nuclear receptors. Biosci Biotechnol Biochem 83: 579-588.

33. Silva AKS, Peixoto CA (2018) Role of peroxisome proliferator-activated receptors in non-alcoholic fatty liver disease inflammation. Cell Mol Life Sci 75: 2951-2961.

34. Lehrke M, Lazar MA (2005) The many faces of PPARgamma. Cell 123: 993-999.

35. Endo-Umeda K, Makishima M (2019) Liver X Receptors Regulate Cholesterol Metabolism and Immunity in Hepatic Nonparenchymal Cells. Int J Mol Sci 20.

36. Li Y, Zalzala M, Jadhav K, Xu Y, Kasumov T, et al. (2016) Carboxylesterase 2 prevents liver steatosis by modulating lipolysis, endoplasmic reticulum stress, and lipogenesis and is regulated by hepatocyte nuclear factor 4 alpha in mice. Hepatology 63: 1860-1874.

37. Liu Y, Chen X, Qiu M, Chen W, Zeng Z, et al. (2014) Emodin ameliorates ethanol-induced fatty liver injury in mice. Pharmacology 94: 71-77.

38. Fu X, Xu AG, Yao MY, Guo L, Zhao LS (2014) Emodin enhances cholesterol efflux by activating peroxisome proliferator-activated receptor-gamma in oxidized low density lipoprotein-loaded THP1 macrophages. Clin Exp Pharmacol Physiol 41: 679-684.

39. Shrimali D, Shanmugam MK, Kumar AP, Zhang J, Tan BK, et al. (2013) Targeted abrogation of diverse signal transduction cascades by emodin for the treatment of inflammatory disorders and cancer. Cancer Lett 341: 139-149.

40. Yang Z, Zhou E, Wei D, Li D, Wei Z, et al. (2014) Emodin inhibits LPS-induced inflammatory response by activating PPAR-gamma in mouse mammary epithelial cells. Int Immunopharmacol 21: 354-360. 
41. Shou X, Zhou R, Zhu L, Ren A, Wang L, et al. (2018) Emodin, A Chinese Herbal Medicine, Inhibits Reoxygenation-Induced Injury in Cultured Human Aortic Endothelial Cells by Regulating the Peroxisome Proliferator-Activated Receptor-gamma (PPAR-gamma) and Endothelial Nitric Oxide Synthase (eNOS) Signaling Pathway. Med Sci Monit 24: 643-651.

42. Kim MO, Park YS, Nho YH, Yun SK, Kim Y, et al. (2016) Emodin isolated from Polygoni Multiflori Ramulus inhibits melanogenesis through the liver $X$ receptor-mediated pathway. Chem Biol Interact 250: 78-84.

43. Huang C, Yang Y, Li WX, Wu XQ, Li XF, et al. (2015) Hyperin attenuates inflammation by activating PPAR-gamma in mice with acute liver injury (ALI) and LPS-induced RAW264.7 cells. Int Immunopharmacol 29: 440-447.

44. Wen Q, Miao J, Lau N, Zhang C, Ye P, et al. (2019) Rhein attenuates lipopolysaccharide-primed inflammation through NF-kappaB inhibition in RAW 264.7 cells: targeting the PPARgamma signal pathway. Can J Physiol Pharmacol.

45. Antonisamy P, Agastian P, Kang CW, Kim NS, Kim JH (2019) Anti-inflammatory activity of rhein isolated from the flowers of Cassia fistula L. and possible underlying mechanisms. Saudi J Biol Sci 26: 96-104.

46. Sheng $X$, Zhu X, Zhang Y, Cui G, Peng L, et al. (2012) Rhein protects against obesity and related metabolic disorders through liver $X$ receptor-mediated uncoupling protein 1 upregulation in brown adipose tissue. Int J Biol Sci 8: 1375-1384.

47. Sheng $X$, Wang $M$, Lu M, Xi B, Sheng $H$, et al. (2011) Rhein ameliorates fatty liver disease through negative energy balance, hepatic lipogenic regulation, and immunomodulation in diet-induced obese mice. Am J Physiol Endocrinol Metab 300: E886-893.

\section{Figures}




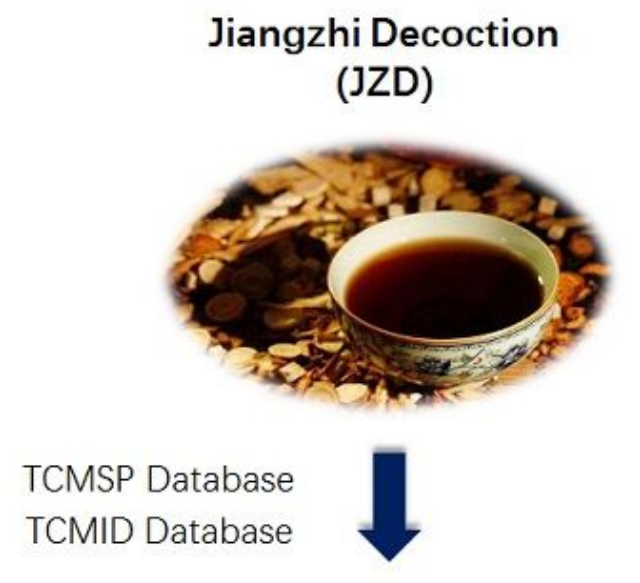

147 Active Ingredients

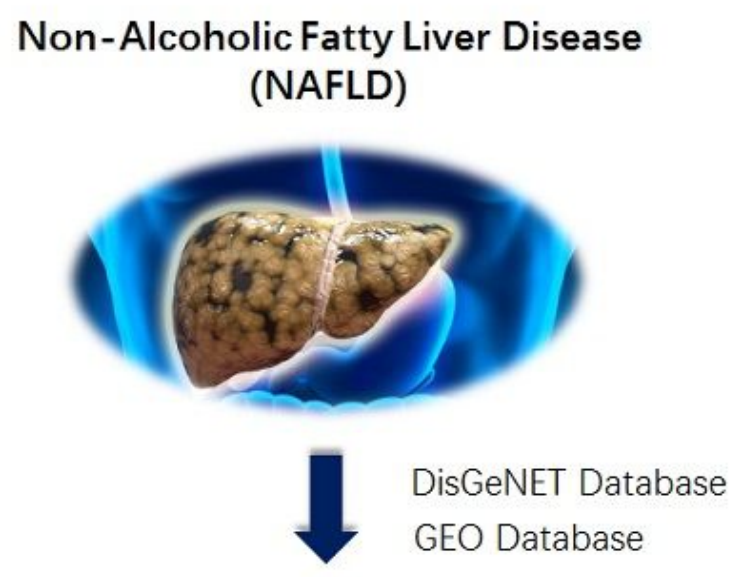

1285 Targets of Active Ingredients

401 NAFLD-Related Genes

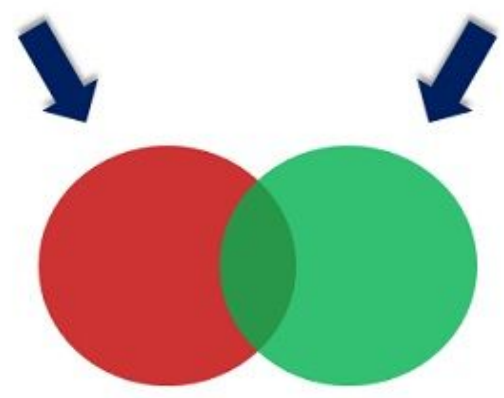

$\downarrow$

59 Overlapped Candidate Targets of JZD against NAFLD

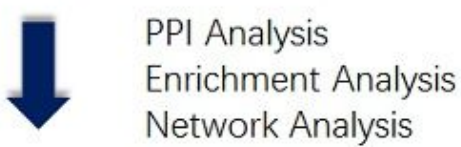

Herbs-Active ingredients-Candidate targets

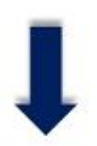

Key Active Ingredients and Core Molecular Mechanisms

Figure 1

The flowchart of the network pharmacology analysis for JZD against NAFLD. 


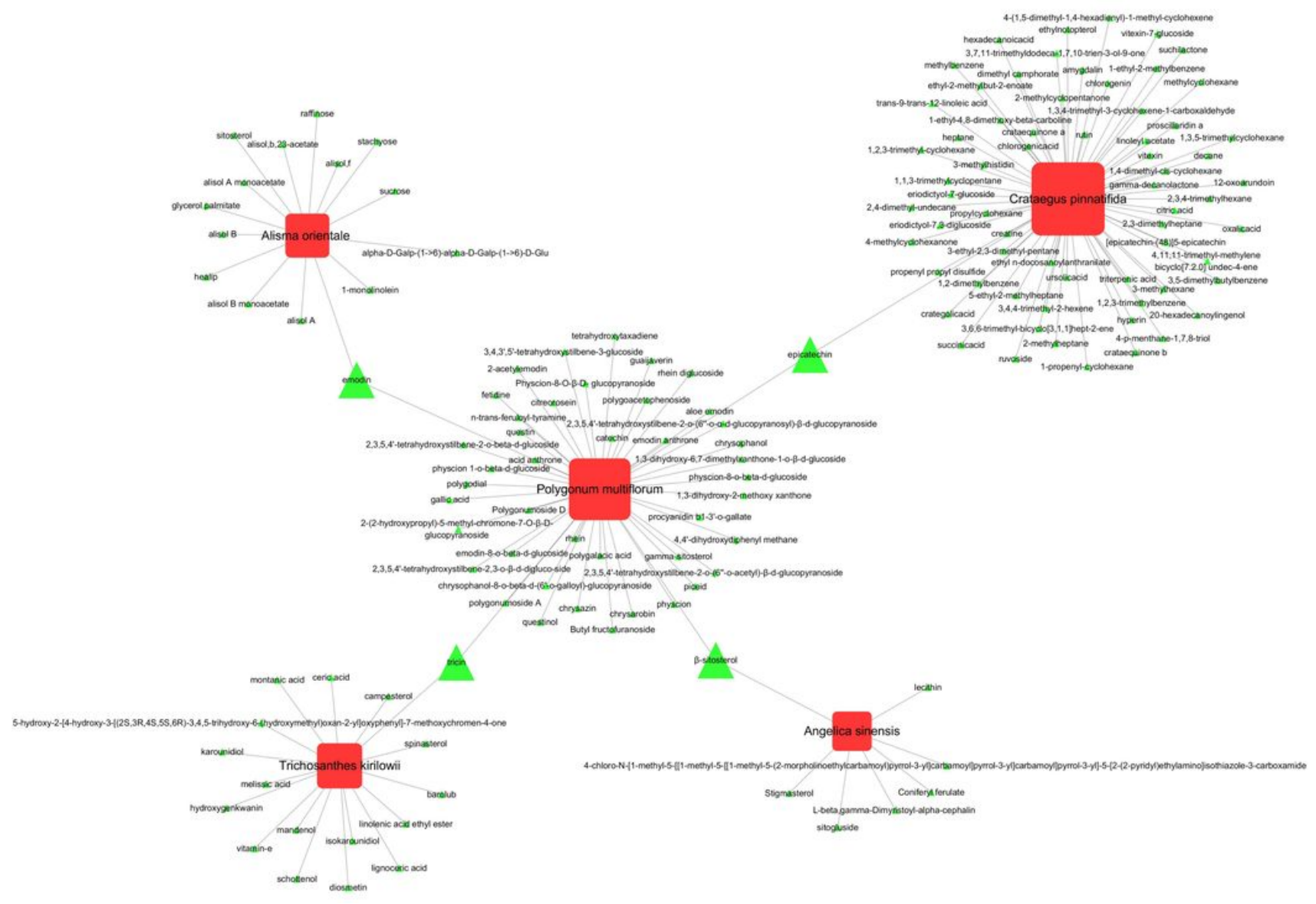

Figure 2

147 active ingredients found in JZD. The red square represents herbs in JZD, and green triangle represents ingredients of those herbs. 


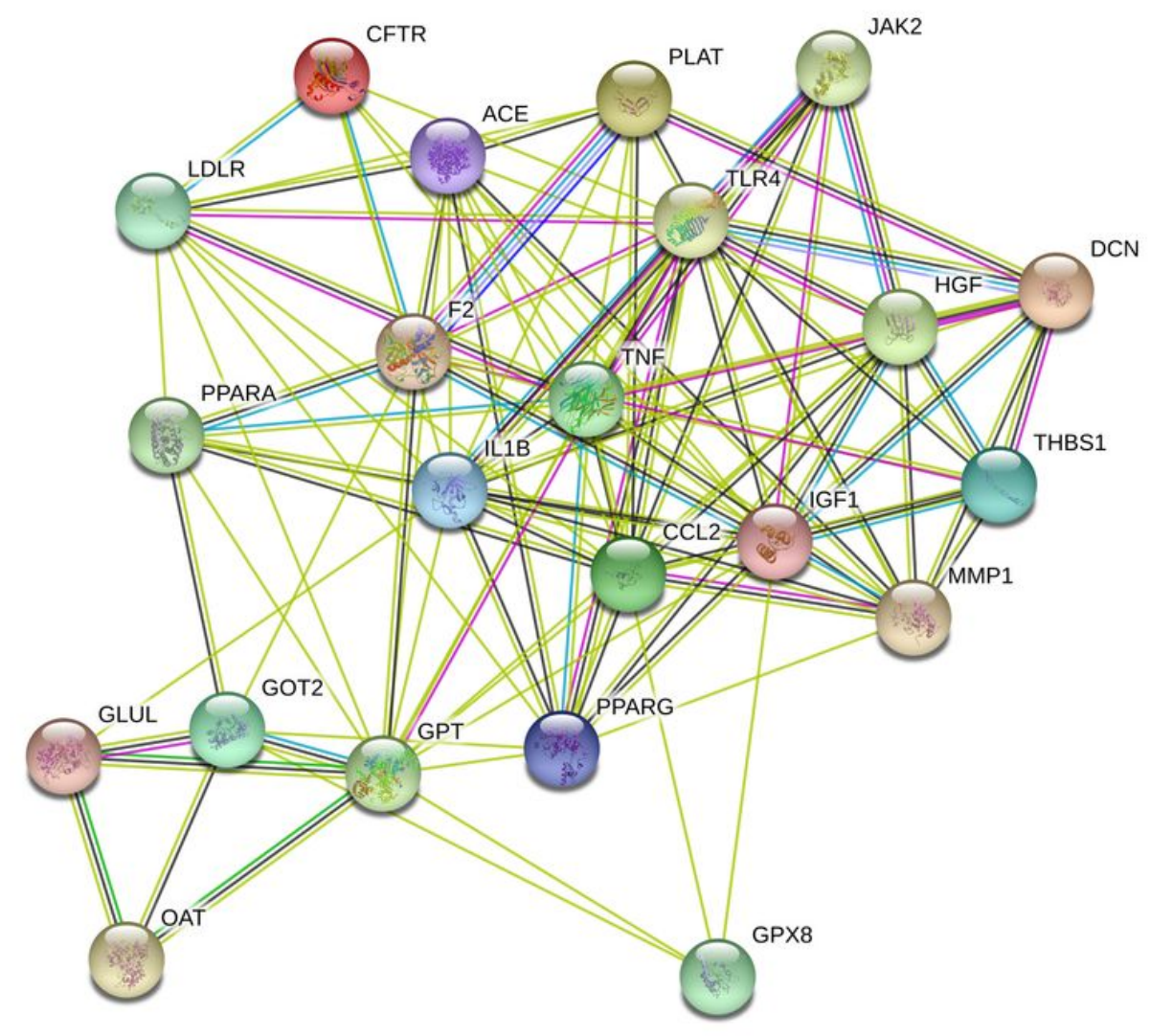

Figure 3

PPI network of 22 core targets of JZD against NAFLD. In the PPI diagram, each solid circle represents a targets, and the middle of the circle shows the structure of the protein. 

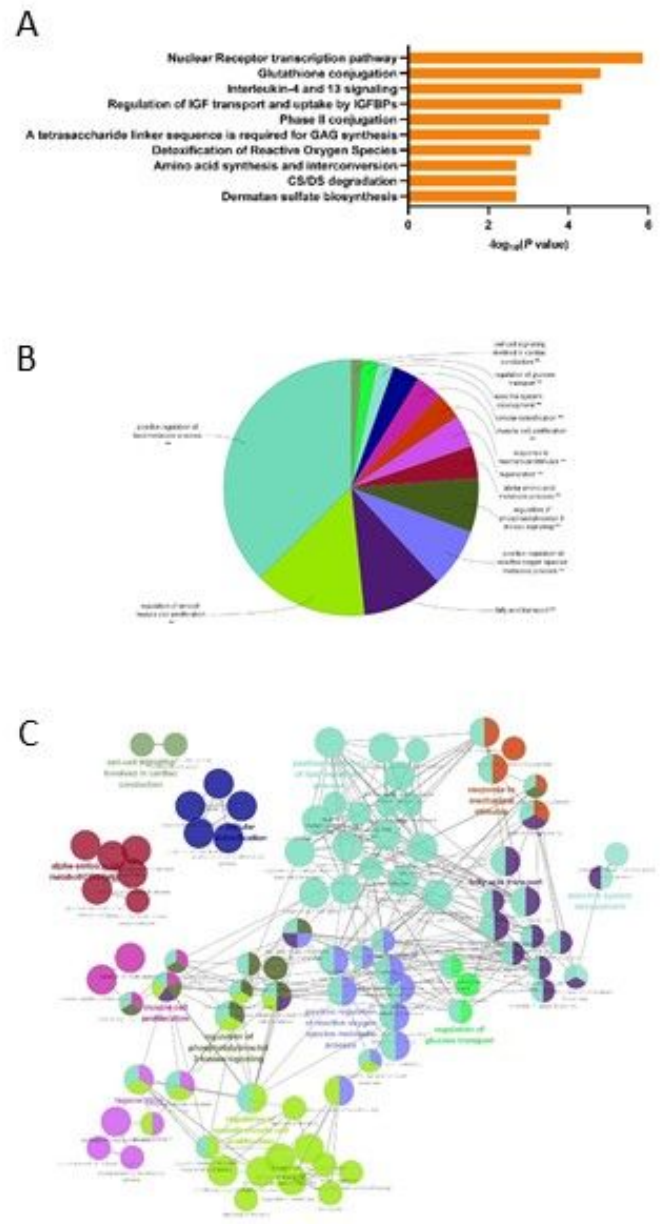

\section{Figure 4}

Functional enrichment analysis from ReactomeFIViz and ClueGO. (A) The column bar graph from ReactomeFIViz. It shows the top 10 significantly related pathways, including nuclear receptor transcription pathway, glutathione conjugation, interleukin-4 and 13 signaling, regulation of insulin-like growth factor (IGF) transport and uptake by insulin-like growth factor binding proteins (IGFBPs), phase II conjugation and so on. (B) The pie chart from ClueGO. It shows the enriched signaling pathway 
categories based on the kappa coefficient, including positive regulation of lipid metabolic process, regulation of smooth muscle cell proliferation, fatty acid transport, positive regulation of reactive oxygen species metabolic process, regulation of phosphatidylinositol 3-kinase signaling, alpha-amino acid metabolic process and so on. (C) The functional enrichment network from ClueGO. The node represents the signaling pathway, and the size of each node represents the enrichment significance of each signaling pathway. The larger the node is, the more significant the pathway is. The line represents the correlation between functions, and the thickness of each line represents the kappa coefficient between functions. The thicker the line is, the greater the kappa coefficient is.
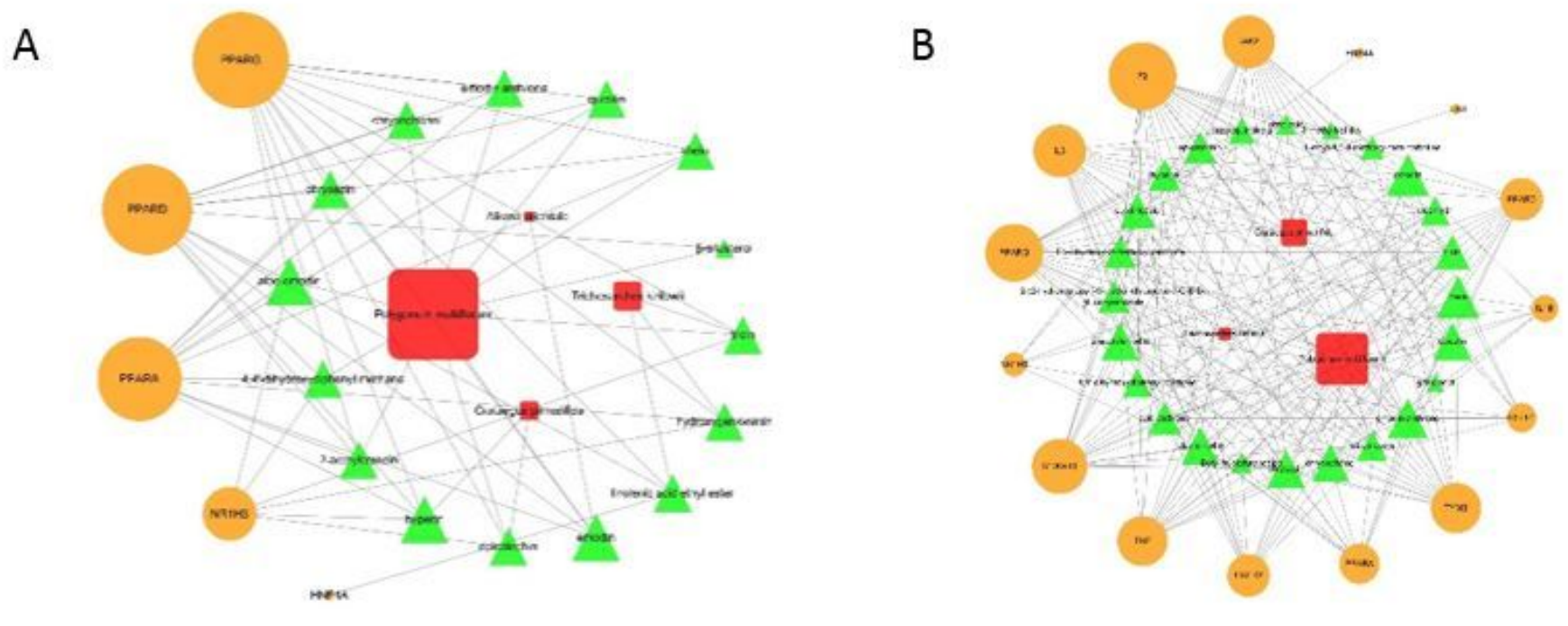

\section{Figure 5}

The top significantly related pathways of "herb-ingredient-target" networks in JZD against NAFLD. (A) Nuclear Receptor transcription pathway; (B) Regulation of lipid metabolic process. The red square represents herbs in JZD, green triangle represents ingredients of those herbs, and orange circle represents candidate targets of JZD against NAFLD. 


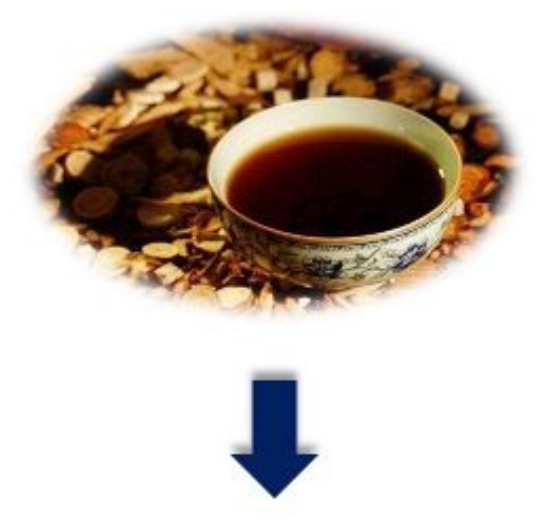

Trichosanthes Kirilowii (TK), Alisma Orientale (AO), Angelica Sinensis (AS), Crataegus Pinnatifida (CP) and Polygonum Multiflorum (PM)

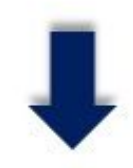

\section{Key Active Ingredients}

Emodin, Emodin anthrone, Hyperin, Questin, Rhein …...

\section{$\downarrow$}

\section{Core Molecular Mechanisms}

Nuclear Receptor Transcription and Lipid Metabolism Regulation

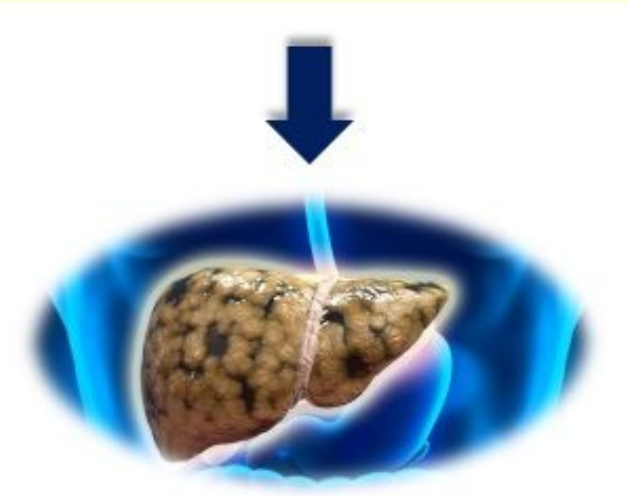

\section{Figure 6}

The key active ingredients and the core molecular mechanisms of JZD against NAFLD. Emodin, emodin anthrone, hyperin, questin, rhein were speculated as the key active ingredients of JZD, and nuclear receptor transcription and lipid metabolism regulation were identified as the core molecular mechanisms of JZD against NAFLD. 


\section{Supplementary Files}

This is a list of supplementary files associated with this preprint. Click to download.

- SupplementaryTable3.xIsx

- SupplementaryTable2.xlsx

- SupplementaryTable1.xlsx 